\title{
Endoscopic ultrasound-guided jejunojejunostomy with rendezvous contrast-filling assistance in afferent limb syndrome
}

A 79-year-old man who previously underwent pancreaticoduodenectomy for distal biliary duct cancer presented with abdominal pain and jaundice. Computed tomography (CT) and magnetic resonance cholangiopancreatography showed dilation of the afferent loop, intrahepatic bile duct, and pancreatic duct, with peripancreatic fat stranding ( $>$ Fig. 1 ), and revealed a recurrent tumor obstructing the biliojejunal anastomotic site.

Drainage was first attempted by percutaneous transhepatic biliary drainage. A subsequent percutaneous attempt with cholangioscopic assistance successfully approached the jejunal limb and enabled catheter placement into the limb, which contained enriched pancreatic juice. Fluoroscopic and CT images revealed that injection of contrast agent showed contrast filling the main pancreatic duct and the jejunal limb was adjacent to the afferent loop (> Fig.2). We then performed a novel procedure using endoscopic ultrasound (EUS) to create an enteric anastomosis with the jejunal limb to drain the pancreatic secretions. Quarter-diluted contrast agent was first pumped into the limb through the percutaneous transhepatic biliary drainage tube to inflate the target cavity. EUS obtained good visualization of the dilated limb from the afferent loop adjacent to the gastrojejunostomy site. The dilated limb was accessed from the afferent loop by an electrocautery-enhanced delivery system, and a 15-mm lumen-apposing metal stent (LAMS) (AXIOS; Boston Scientific, Marlborough, Massachusetts, USA) was deployed in a single-step freehand fashion. The LAMS was then successfully bridged as a jejunojejunostomy ( Fig.3). Finally, biliary metal stents were deployed percutaneously for biliary endoprosthesis ( $\triangleright$ Video 1 ).
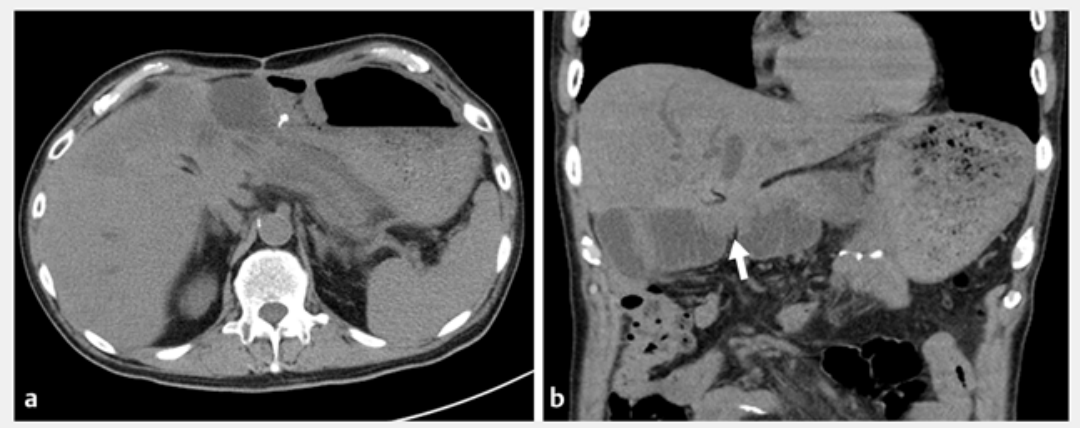

- Fig. 1 a, b Axial computed tomography image and coronal reconstruction image show a dilated jejunal limb with dilated intrahepatic bile duct and pancreatic duct along with peripancreatic fat stranding, and reveal a recurrent tumor (arrow) obstructing the biliojejunal anastomotic site.
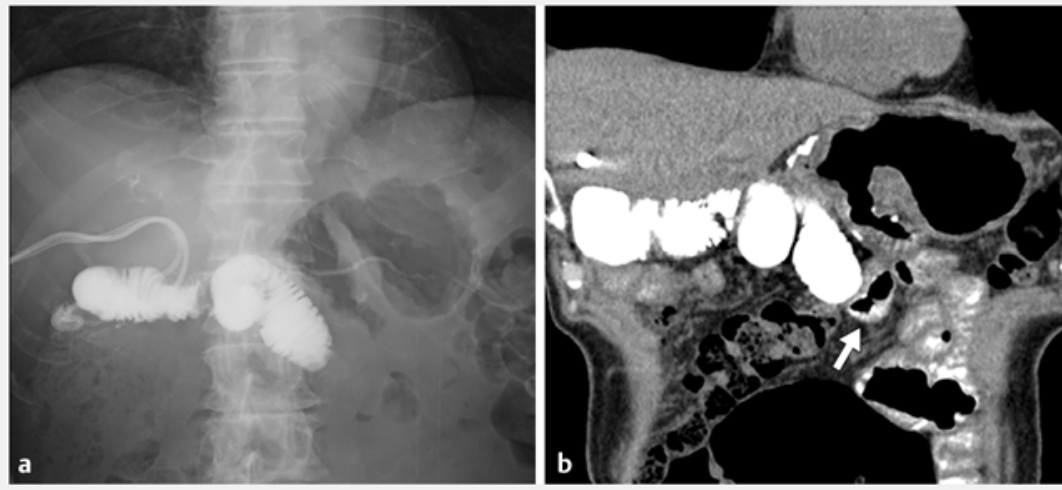

Fig. 2 a Fluoroscopic image showing contrast agent injected via a percutaneous transhepatic tube positioned in the jejunal limb. The distended jejunal limb and contrast-filled main pancreatic duct are apparent. b Coronal computed tomography reconstruction image showing the location of the jejunal limb adjacent to the afferent loop (arrow).

As an alternative treatment in palliative surgical bypass for malignant afferent limb syndrome, the feasibility of EUSguided transgastric drainage with a LAMS has been reported in a few cases [1-3] but is considered a high-risk option. Prior to EUS-guided drainage, rendezvous pumping with diluted contrast agent into the jejunal limb facilitated better visualization on EUS and fluoroscopic images and safer deployment of the LAMS. Rendezvous pumping appears to be feasible as a low-risk technique.

Endoscopy_UCTN_Code_TTT_1AR_2A 


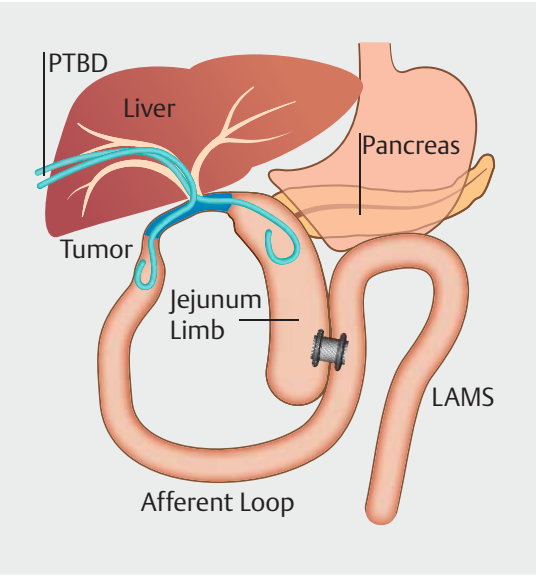

- Fig. 3 Schematic illustration of the present case of malignant afferent limb obstruction. Endoscopic ultrasoundguided jejunojejunostomy was performed with contrast-filling assistance and a lumen-apposing metal stent. LAMS, lumen-apposing metal stent; PTBD, percutaneous transhepatic biliary drainage.

\section{Competing interests}

The authors declare that they have no conflict of interest.

The authors

Michihiro Yoshida ${ }^{1}$, Hiroyuki Imafuji², Kazuki Hayashi ${ }^{1}{ }^{\complement}$ Itaru Naitoh ${ }^{1}$, Yasuki Hori ${ }^{1}$, Akihisa Kato', Yoichi Matsuo ${ }^{2}$

1 Department of Gastroenterology and Metabolism, Nagoya City University Graduate School of Medical Sciences, Nagoya, Japan

2 Department of Gastroenterological Surgery, Nagoya City University Graduate School of Medical Sciences, Nagoya, Japan
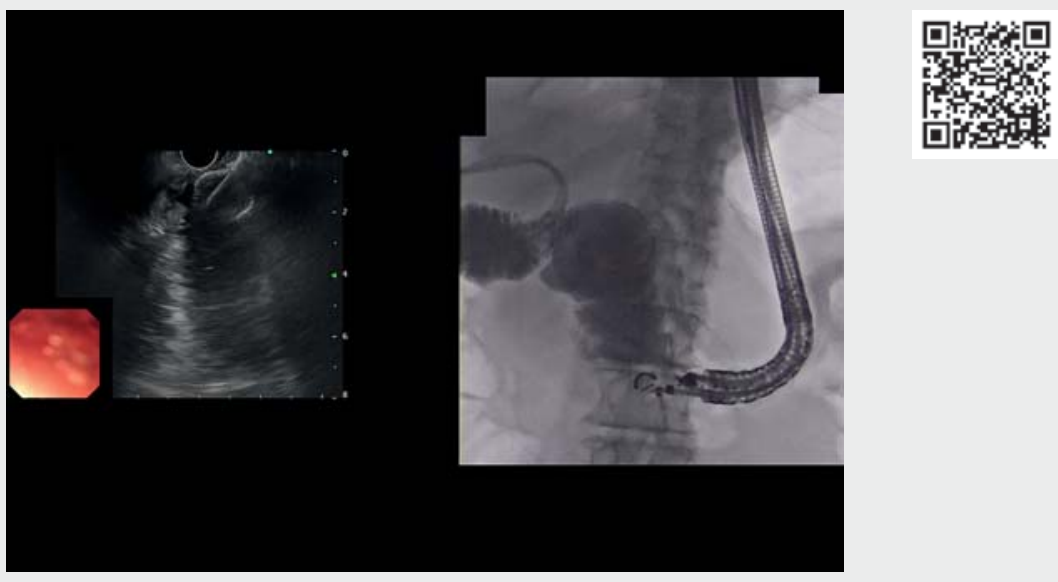

$\checkmark$ Video 1 Novel technique for endoscopic ultrasound-guided jejunojejunostomy with rendezvous luminal contrast-filling assistance in malignant afferent limb syndrome.

\section{Corresponding author}

\section{Kazuki Hayashi}

Department of Gastroenterology and Metabolism, Nagoya City University Graduate School of Medical Sciences, 1 Kawasumi, Mizuho-cho, Mizuho-ku Nagoya 467-8601, Japan

khayashi@med.nagoya-cu.ac.jp

\section{References}

[1] Shah A, Khanna L, Sethi A. Treatment of afferent limb syndrome: novel approach with endoscopic ultrasound-guided creation of a gastrojejunostomy fistula and placement of lumen-apposing stent. Endoscopy 2015; 47: E309-E310

[2] Lakhtakia S, Chavan R, Basha J et al. Endoscopic ultrasound-guided transjejunal drainage of an obstructed afferent loop using a novel lumen-apposing metal stent. Endoscopy 2019; 51: E253-E254

[3] Wang G, Liu X, Wang S et al. Endoscopic ultrasound-guided gastroenterostomy: a promising alternative to surgery. J Transl Int Med 2019; 7: 93-99

\section{Bibliography}

Endoscopy 2022; 54: E49-E50

DOI 10.1055/a-1348-0707

ISSN 0013-726X

published online 5.3.2021

(C) 2021. Thieme. All rights reserved.

Georg Thieme Verlag KG, Rüdigerstraße 14, 70469 Stuttgart, Germany

\section{ENDOSCOPY E-VIDEOS \\ https://eref.thieme.de/e-videos}

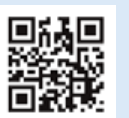

Endoscopy E-Videos is an open access online section, reporting on interesting cases and new techniques in gastroenterological endoscopy. All papers include a high quality video and all contributions are freely accessible online. Processing charges apply (currently EUR 375), discounts and wavers acc. to HINARI are available.

This section has its own submission website at https://mc.manuscriptcentral.com/e-videos 